\title{
Headache in the Emergency Room
}

\author{
Kourosh Kahkeshani and Huma U Sheikh
}

Mount Sinai Hospital, New York, NY, US

$\mathrm{H}$ eadache is seen in the emergency room (ER) on a daily basis and is a vague symptom, which can be a part of many different types of conditions and diseases. Although most people who come to the ER will have a benign headache, it is important to make sure that dangerous causes of headache are ruled out. There are a number of features that may alert someone to a secondary cause of headache. This article highlights two specific secondary headache syndromes, including carotid dissection and reversible cerebral vasoconstriction syndrome. It also broadly points out other possible causes of secondary headache. The last section focuses on a common primary headache that presents to the ER, migraine and its treatment.

\section{Keywords}

Secondary, emergency room, headaches, SNOOP, migraine

Disclosure: Kourosh Kahkeshani and Huma U Sheikh have nothing to declare in relation to this article.

Authorship: All named authors meet the International Committee of Medical Journal Editors (ICMJE) criteria for authorship of this manuscript, take responsibility for the integrity of the work as a whole, and have given final approval to the version to be published.

open Access: This article is published under the Creative Commons Attribution Noncommercial License, which permits any noncommercial use, distribution, adaptation, and reproduction provided the original author(s) and source are given appropriate credit.

Received: January 4, 2017

Accepted: April 4, 2017

Citation: US Neurology, 2017;13(2):94-8

Corresponding Author: Huma U Sheikh, Mount Sinal Hospital-Downtown, 10 Union Square, New York, NY 10003, US. E: hsheikh@chpnet.org

\section{Identifying secondary headaches in the emergency room}

In 2008 alone (according to most recent data), there were over 3 million visits to the emergency room (ER) where headache was the primary complaint, in the US. ${ }^{1}$ Headache comprised a little over $2 \%$ of all ER visits, making it one of the most common neurological complaints in the ER. ${ }^{1}$

When all the reasons are tallied, the most common etiology of headache was a primary headache diagnosis; of which migraine was the most common. ${ }^{1}$ However, when someone presents to the ER with a headache, it is imperative for the physician to rule out more dangerous or life-threatening causes of headaches. Therefore, it is important to have a systematic approach to evaluating a patient with headache. One of the first steps in the ER when treating a patient with headache is to obtain a thorough history and conduct a physical assessment in order to identify red flags and rule out secondary causes of the disorder. Some of the important elements to discern include determining if there is a history of primary headache syndrome, along with the patient's family and medical history and past medication use. ${ }^{2}$ It is also vital to characterize the headache in detail, including build up of severity, location, associated symptoms such as migrainous or autonomic features, and exacerbating or alleviating factors. A change in headache characteristics that deviate from a patient's baseline headache may allude to a new underlying process and possibly provide insight into secondary causes of headache. ${ }^{3}$ Certain features in the patient's history can point to a specific etiology, although it is also important to consider a wide range of differential diagnoses so that life-threatening conditions are not overlooked.

A mnemonic that can help to determine if there may be a secondary cause for headache is "SNOOP." The "S" stands for systemic, including presence of fever, weight loss or other pain, including body and muscle pain. The " $\mathrm{N}$ " stands for neurological, or cases in which there are other neurological signs with headache, including a focal sign on physical examination. The "O" stands for onset, which includes a sudden onset of a severe headache, and is widely referred to as the "thunderclap." A thunderclap headache is one that comes on suddenly, within seconds, and is severe from onset. In general, these types of headache are a cause for concern. The second "O" stands for older age; usually the first onset of a headache in someone older than 40 needs further workup. The " $\mathrm{P}$ " refers to a change in pattern, with a prior history of primary headaches, worsening of the severity or frequency can be a sign of a secondary cause. ${ }^{4}$

Other concerning signs are unilateral or "side-locked" headaches that may point to a mass, lesion, or stroke. Headaches that are associated with visual changes or tinnitus may point to disorders causing increased intracranial pressure. Patients who are immunocompromised, including those with 
a malignancy or with HIV should also have further testing. Every patient presenting to the ER requires a complete neurological exam, including an ophthalmologic examination, with careful assessment for papilledema or other subtle neurological signs, especially unilateral findings., ${ }^{2,5}$

The workup for a headache will be based on the possible differential diagnosis. Although most people will receive at least some type of initial imaging, the American Academy of Neurology recommends further testing in patients with an increase in frequency of headaches or with an abnormal examination, or headaches that awaken a person from sleep. ${ }^{6}$ Computed tomography (CT) is superior for identifying acute blood products, such as hemorrhage, while magnetic resonance imaging (MRI) is more sensitive for posterior fossa abnormalities, meningeal disease, and disorders of cerebrospinal pressure. ${ }^{5-6}$ If there is concern for an infection or inflammatory process, or disorders related to cerebrospinal pressure, a LP is warranted. If there is clinical suspicion for disorders related to the vessels, magnetic resonance angiography (MRA) or magnetic resonance venography (MRV) are the most sensitive and specific imaging tools. ${ }^{6}$

Headaches during pregnancy require special attention, since there are hormonal and hematological changes that increase the risk of certain disorders. The workup for headache in a pregnant woman would be similar to that of a non-pregnant woman, with a few considerations. If imaging is required, MRI is preferred over $\mathrm{CT}$ scans since there is no risk of radiation. Contrast is usually considered safe, categorized as level $\mathrm{C}$ by the US Food and Drug Administration (FDA), although iodinated contrast does carry the risk of decreasing thyroid function in the newborn. ${ }^{7}$ It is also important to keep the developing fetus in mind when deciding on a treatment plan.

\section{Secondary headaches}

There are a number of diseases in which the main presenting symptom can be headache. Although many of these will be easily discerned by other signs and symptoms, there are also a few disorders that need to be approached with a high degree of suspicion, since headache may be the only prominent feature. A useful way to classify secondary headaches is: vascular, infectious, inflammatory, traumatic, neoplastic, and toxic. Although this is not a comprehensive list, these are some of the possible disorders to keep in mind in patients presenting with headache.

\section{Vascular}

Some common diseases like stroke and intracranial hemorrhage can present with a headache. Headache is a common feature of hemorrhage, with headache present in up to $50 \%$ of patients with intracranial hemorrhage $(\mathrm{ICH}){ }^{8}$ Posterior fossa infarcts are more likely to present with headache than those in the anterior region. ${ }^{8-9}$ In these cases, there are usually other focal signs and symptoms that point to this diagnosis. subarachnoid hemorrhage, (SAH) which is most often caused by trauma or an aneurysm, presents as a very severe, acute-onset headache, usually regarded as the "worst headache" or "thunderclap."10-11 SAH usually presents with other neurologic findings such as loss of consciousness, vomiting, seizures, or meningismus. ${ }^{12}$ Headache prior to $\mathrm{SAH}$, presumably from sentinel bleeding, may precede the hemorrhage by up to 20 days in up to $43 \%$ of patients. ${ }^{13}$ Given the high morbidity and mortality of SAH, suspicion should prompt immediate cranial neuroimaging. It is important to note that sensitivity of CT for diagnosing SAH declines substantially over days following the initial headache. LP is usually done, while computed tomography angiopgraphy (CTA) or angiography is considered gold standard and should be performed to rule out SAH if suspicion of SAH is maintained. ${ }^{12}$

Amyloid angiopathy, hypertensive hemorrhage, and vascular malformations are some of the most common causes of $\mathrm{ICH}$. Patient stabilization, aggressive blood pressure management, reversal of any coagulopathies, and pre-operative assessment should be part of the management. Aneurysms that have not ruptured but are large enough to cause mass effect can also present with headache. This is similar to other vascular malformation, including cavernomas or arteriovenous malformations $(A V M)$, that can cause headache through mass effect or if they rupture. Although there is variation, cavernomas most often present with seizures while AVM have headache as a more dominant feature. ${ }^{14}$

Reversible cerebral vasoconstrictive syndrome (RCVS) is a clinical syndrome where the hallmark symptom is a sudden-onset severe or "thunderclap" headache. In the past, it has gone under-recognized but is becoming more widely reported recently. The headache is described as severe, diffuse, and reoccurring over 7-10 days, and, although symptoms are usually self-limiting, complications can occur, including subarachnoid hemorrhage, intracerebral hemorrhage, stroke, and even death from "fulminant" vasoconstriction..$^{15-17}$ Females are more likely to be affected, especially those who are post-partum, and several triggers have been identified, including immunosuppressants, cytotoxic agents, recent neurosurgical procedures non-aneurysmal $\mathrm{SAH}$, and vasoactive substances, such as marijuana, cocaine and heroine. ${ }^{15,16,18}$ Migraine is thought to be a risk factor but there is usually an alternate precipitating factor. The diagnostic algorithm is similar to that for SAH, including CT and LP to rule out hemorrhage, while the gold standard is angiogram, which may show areas of large- and medium-sized arterial vasoconstriction; MRA is also an acceptable alternative. ${ }^{16}$ Calcium-channel blockers, such as nimodipine or verapamil, are the primary treatment, although no controlled studies have been performed to confirm their efficacy. ${ }^{19}$

Dissection, which is a tear in the lining of an artery, is another clinical syndrome where headache is a prominent feature. Vessels can dissect anywhere along the cervicocephalic vasculature, and it may occur spontaneously or following trauma, and may be complicated by stroke, intracerebral hemorrhage, or aneurysmal formation. ${ }^{20-21}$ Patients nearly always present with headache and/or neck pain. The classic triad is described as headache, Horner's syndrome and cerebral ischemia, including transient ischemic attacks (TIAS), which are present in about $30 \%$ of cases. ${ }^{22}$ Vertebral arterial dissection may present with occipital headaches and symptoms suggestive of brainstem or posterior circulation infarction. ${ }^{23}$ Vascular imaging is paramount in diagnosis, and although digital subtraction angiography is considered the gold standard, vascular imaging with CTA or MRA are both acceptable techniques in the emergency department setting. ${ }^{24}$ Antiplatelet therapy has been shown to be as effective as anticoagulation in treatment, and endovascular stabilization may be warranted in some cases. ${ }^{25}$

Cerebral venous sinus thrombosis (CVST) is a condition where a clot can develop anywhere along the venous system in the central nervous system (CNS). It almost always presents with headache and is often accompanied with blurred vision and signs of raised intracranial pressure (ICP) such as optic disc edema, nausea, or vomiting. More dangerous symptoms from increased intracranial pressure include decreased 
level of consciousness, encephalopathy, focal neurologic deficits, and seizures. ${ }^{26-27}$ Headache features in CVST are usually acute, severe, and unilateral, but can vary from diffuse and progressive to "thunderclap" in description. ${ }^{28-29}$ Trauma, infectious causes, inflammatory diseases, use of oral contraceptives, and prothrombotic conditions, including pregnancy, are all potential risk factors. ${ }^{27}$ Both combinations of CT with CT venogram or MRI with magnetic resonance venogram can be used to confirm the diagnosis. ${ }^{30}$ Treatment entails systemic anticoagulation, even if there is evidence of cranial hemorrhage. ${ }^{27}$ Symptomatic treatment can be offered for treatment of headaches.

Posterior reversible encephalopathy syndrome (PRES) was first described in 1996. It is seen in clinical conditions that increase the permeability of the blood-brain barrier, especially vulnerable arteries in the posterior regions of the brain. ${ }^{31}$ In a mixed prospective and retrospective analysis of 120 patients with PRES, Fugate et al. found acute hypertension (86\%) and seizures (74\%) to be the most common presenting features, with headache (26\%), encephalopathy (28\%), and visual changes (20\%) being less common. ${ }^{32}$ Risk factors for developing PRES include concurrent immunosuppression therapy, existing hypertension, infection/sepsis, autoimmune conditions, as well as preeclampsia and eclampsia. ${ }^{31}$ Brain MRI usually shows T2-hyperintensity vasogenic edema of cortical and subcortical white matter, usually involving, but not exclusively, the posterior regions of the brain. ${ }^{31}$ Treatment involves aggressive control of blood pressure, and addressing any related co-morbid conditions, e.g., removal of offending immunosuppressive agents. It can also be a complication of preeclampsia, in which case, delivery of the child can be helpful. ${ }^{33}$

\section{Infectious/inflammatory}

There are potentially many types of infections where headaches may be a symptom. The meninges, that is, the dural covering of the brain, can be infected by bacterial, viral or fungal pathogens causing a meningitis, which classically presents with the triad of fever, headache, and stiff neck. ${ }^{10}$ Diagnosis is made by LP and cerebrospinal fluid (CSF) examination; however, empiric broad spectrum antibiotics should be initiated immediately, since delay in treatment may be fatal. New chronic headaches with fever and focal neurologic findings, especially in the setting of immunosuppression, should raise the suspicion for cerebral abscess or subdural empyema. ${ }^{5}$ Imaging and LP can be helpful in diagnosis and antibiotics/antifungals are usually the first line of treatment. In patients with HIV, infectious causes, including cryptococcol meningoencephalitis, may cause headache. Extracranial infectious such as sinusitis, otitis, or dental abscesses may also cause headache via referred pain. Therefore, it is important to keep a wide differential and take all symptoms into account.

Other less common inflammatory causes of headache include inflammation of the arterial structures, i.e., a vasculitis. Vasculitis can be part of a systemic disease or isolated to the CNS. ${ }^{34}$ In vasculitis, some of the symptoms include headache, encephalopathy, and even cerebral infarcts, although diagnosis is often challenging to the clinician. ${ }^{35}$ In older women, who present with more subacute headaches, giant cell arteritis is a type of vasculitis that should be ruled out. Giant cell arteritis (GCA), or temporal arteritis, is a chronic vasculitis of large- and medium- sized vessels, that affects, almost exclusively, the elderly population, with mean age of presentation of 65 years or more. ${ }^{36}$ Persistent headache, either unilateral or bilateral, with jaw claudication are hallmark symptoms, while patients may also present with fever and even acute vision loss. ${ }^{36}$ Elevated inflammatory markers, erythrocyte sedimentation rate (ESR) or C-reactive protein (CRP), can be valuable tools in diagnosis, but negative values do not rule out GCA. ${ }^{35}$ Diagnosis may be aided by neuroimaging, either ultrasonography or MRI, and a temporal artery biopsy may also be done for a definitive diagnosis. ${ }^{36}$ Immediate initiation of treatment with prednisone or prednisolone is recommended if GCA is suspected. ${ }^{35}$ Other cerebral vasculitides include Takayasu's arteritis, polyarteritis nodosa, Churg-Strauss syndrome, Behçet's disease, and primary angiitis of the CNS (PACNS).

\section{Traumatic}

Traumatic injury to the brain or skull is a major contributor for secondary headaches encountered in the emergency department. ${ }^{37} \mathrm{CT}$ of the head and neck can help identify potentially life-threatening fractures or hemorrhages. However, occult pathologies, such as carotid or vertebral dissection, may be overlooked, and a thorough history and neurologic examination should be performed. If suspected, more invasive vascular imaging may be indicated. Treatment in acute trauma should primarily be geared toward stabilizing the patient and to monitor for signs of rising ICP, such as cranial nerve palsies. ${ }^{38}$ Intraparenchymal (non-aneurysmal) subarachnoid hemorrhage, cerebral contusion and subdural/epidural hematoma are all complications that may occur. Patients may also develop chronic headaches, even from mild concussions. They may be of any phenotype, including headaches similar to migraines or tension-type headaches in character.

\section{Neoplastic/cerebrospinal fluid pressure}

Intracranial neoplastic processes can be due to primary CNS tumors, but are usually secondary to other systemic malignancies; most commonly gastrointestinal, lung, breast, renal, and melanoma. Classically, patients complain of early morning headaches with nausea and vomiting that are either de novo or different than their own baseline headaches, if they previously suffered from headaches. ${ }^{39}$ Headache symptoms are often relieved with corticosteroid therapy or anti-emetics, but treatment of the tumor may ultimately relieve the headaches. ${ }^{39}$

Alterations of intracranial CSF pressure, either elevated in intracranial hypertension or reduced in intracranial hypotension, can present with specific headache phenotypes. Idiopathic intracranial hypertension $(I I H)$, formerly known as pseudotumor cerebri, occurs classically in young, obese women, and headaches can be associated with nausea, vomiting, or visual field disturbances.$^{40}$ Headache is nearly always present, and papilledema is suggestive of diagnosis. ${ }^{40-41}$ As the name implies, the process is idiopathic and diagnosis is based on exclusion of other causes of raised ICP such as mass lesions or medication-induced pseudotumor cerebri. LP is routinely performed for diagnosis, with CSF pressure $>20 \mathrm{~cm} \mathrm{H}_{2} \mathrm{O}$ as diagnostic, or $>25 \mathrm{Cm} \mathrm{H}_{2} \mathrm{O}$ in obese patients. Rare reports of tonsillar herniation, however, have been reported after LP in patients with $\mathrm{IH} .{ }^{41}$ Patients are treated with oral diuretics, such as acetazolamine or topiramate, as well as being referred to an ophthalmologist for formal visual field testing.

Conversely, Iow CSF pressure, i.e., intracranial hypotension, usually occurs after trauma to the dura following neurosurgical procedures, LP or spinal epidurals. It may also occur spontaneously due to a multitude of etiologies, including spinal meningeal diverticula or connective tissue diseases like Marfan's syndrome. ${ }^{42}$ Headaches associated with low CSF pressure are classically described as postural orthostatic headache, in which the headache improves dramatically in the supine position and 
may or may not be accompanied by other symptoms such as nausea, vomiting, visual field changes, or tinnitus. ${ }^{42}$ At times, the diagnosis can be challenging and involves neuroimaging and LP, although high suspicion with negative initial testing may warrant more advanced and invasive diagnostic imaging techniques. ${ }^{43}$ Initial treatment of low CSF pressure headaches in the ER setting are geared toward symptom relief and correcting the insulting etiology. Intravenous (IV) or oral caffeine and IV fluids are often used, but no randomized controlled studies have been conducted to support their use. ${ }^{44}$ An epidural blood patch may be attempted if conservative treatment fails, and if localized CSF leak is identified, corrective surgery may be warranted.

\section{Toxic/metabolic/anatomic}

There are a number of toxic and metabolic issues that can also present with headaches, in addition to other signs and symptoms. Carbon monoxide poisoning is an important one to keep in mind, along with a list of medications where headache may be a side effect, either as part of treatment or as a withdrawal symptom. ${ }^{45}$ Caffeine withdrawal can cause acute headaches along with dehydration or low glucose levels, for example, from fasting. Headache is also a common symptom of persistent or acute high blood pressure, referred to as hypertensive urgency or emergency.

A large variety of prescription and over-the-counter (OTC) medications may induce headaches, and detailed discussion is beyond the scope of this article. Clinicians should be suspect of any newly initiated medications that may cause secondary headache. If discovered, offending medications may or may not be changed or discontinued in the ER and any changes should be communicated to the original prescribing physician. Medicationoveruse headache $(\mathrm{MOH})$ is a common, well-described entity that develops in patients with underlying primary headache syndromes. As the name suggests, MOH occurs after chronic use (usually months) of OTC or prescription headache relievers, such as nonsteroidal anti-inflammatory drugs (NSAIDS), narcotics or triptans. ${ }^{46}$ It is important to note that lesions or changes to the anatomy of the head and neck may cause headache through referred pain. Conditions like acute angle closure glaucoma and trigeminal neuralgia often have face and head pain as part of the presenting symptoms.

Due to physiologic changes that occur during pregnancy, gravid women require special consideration in the evaluation of headache. Preeclampsia and eclampsia can occur in pregnancy, usually after the 20th week of gestation, but may even occur in the post-partum period. Along with headache as a common presenting symptom, the two other diagnostic signs, hypertension and proteinuria, are usually present. The addition of seizures or coma indicate eclampsia, and this is considered a medical emergency, where urgent delivery of the baby may be indicated. ${ }^{47}$ Several other secondary headache syndromes discussed above are prevalent in the peri-partum population, including posterior reversible encephalopathy syndrome, sinus venous thrombosis, post-epidural headache, CVST, and Sheehan's syndrome and post-epidural headaches.

\section{Treatment}

The treatment for secondary headaches, for the most part, will depend on the underlying condition, and if there is additional pain therapy required, it is usually on the phenotype of the headache that is present. This usually requires abortive therapy and, sometimes, the use of more long-term preventive therapy. Abortive therapy refers to acute treatment of pain at the time of headache, while preventive therapy generally involves daily medication to help prevent the onset of headaches. Treatment choices for both abortive and preventative therapy is limited to pregnancysafe medications; however, a plethora of therapeutic options exist in this population.

Although it is important to rule out dangerous causes of headaches, most people who present to the ER will likely have an exacerbation of a primary headache sydnrome. Of these, the most common is migraine. By the time a person comes into the ER, they have likely tried at least a couple of pain medications that have not worked to resolve a headache. A migraine that lasts more than 24 hours is typically more difficult to treat and after 48 hours the headache is described as status migrainosus. ${ }^{48}$ The main strategy to treating migraines in the ER involves providing hydration and adequate pain control as well as treating the other associated features like nausea, for example. ${ }^{48}$

Medications used to treat acute migraine attacks include both oral and IV medications. Unfortunately, no set protocol exists; and medications are generally prescribed based on physician preference or comfort level. Recently, the American Headache Society published a systematic review of pharmacotherapy for acute migraine to guide first-line treatment in the ER and to determine whether corticosteroids prevented recurrence of migraine in patients discharged from the ER. ${ }^{49}$

The researchers identified all randomized studies comparing injectable medications to either placebo or control for the treatment of acute migraine in adults. Tiered recommendations for use of medications in the ER setting were developed based on strength of the study and the medication's efficacy and side effect profile. ${ }^{49}$ Despite not offering Level A, or must offer, recommendation for any drug, the authors determined that IV metoclopramide, prochloperazine and subcutaneous sumatriptan were in the category of should offer (Level B) to patients as first line if no contraindications exist. ${ }^{49}$ If these medications are contraindicated, Level C-backed evidence, or may offer, recommends the use of IV acetaminophen, acetylsalicylic acid, dexketoprofen, diclofenac, ketorolac, valproate, and parenteral chlorpromazine, droperidol, or haloperidol. Dexamethasone was not recommended for the treatement of acute migraine, however, the authors suggest treatment with dexamethasone for prevention of migraine recurrence with Level B recommendation. ${ }^{49}$ Narcotics, such as morphine and hydromorphone, are not recommended due to lack of their evidence in regards to efficacy, as well as concerns of the potential for dependence or addiction. ${ }^{49}$

For pregnant women, the use of triptans is controversial, along with NSAIDS, which can cause miscarriage or malformation. ${ }^{50}$ Although acetaminophen has long been considered safe for use in pregnancy, a new study has linked its use in pregnancy to risk of attention deficit hyperactivity disorder (ADHD), where the effect appears to be related to quantity of acetaminophen used. ${ }^{51}$ Both NSAIDs and triptans are safe in lactation, with the recommendation that women should pump 2-4 hours following triptan ingestion and discard the milk..$^{52}$ The two safest preventives in pregnancy include biofeedback and nerve blocks. ${ }^{52}$ Magnesium can be used as an abortive, daily as a preventive or in intravenous form as acute treatment in the ER. The Hales Lactation Rating and Briggs Category are useful resources when choosing therapies during pregnancy. 


\section{Conclusion}

Overall, the goal when encountering a patient in the ER with headache is to quickly and accurately determine the underlying etiology, as well as effectively controlling the patient's pain. In order to do this, a thorough and complete history and physical is key. After secondary causes are ruled out, the treatment for headache will depend on its phenotype and other associated symptoms. Headache in the ER has a wide differential, therefore a systematic and organized approach is required. $\square$
1. Lucado J, Paez K, Elixhauser A, Headaches in U.S. hospitals and emergency departments, 2008, HCUP Statistical Brief \#111 2011. Agency for Healthcare Research and Quality, Rockville, MD. Available at: https://www.hcup-us.ahrq.gov/reports/statbriefs/ sb111.pdf (accessed April 13, 2017)

2. Digre KB, Headaches during pregnancy, Clin Obstet Gynecol, 2013;56:317-29.

3. Von Wald T, Willing AD, Headache during pregnancy, Obstet Gynecol Surv, 2002:57:179-85.

4. Dodick DW, Pearls: headache, Semin Neurol, 2010;30:74-81

5. Dodick DW, Clinical cues and clinical rules: Primary vs secondan headache, Adv Stud Med, 2003;3:S550-55.

6. Morey SS, Headache consortium releases guidelines for the use of CT or MRI in migraine work up [Pract Guide], Am Fam Physician, 2000;62:1699-701.

7. Mandel $\mathrm{S}$, Hemiplegic migraine in pregnancy, Headache 1988;28:414-6

8. Vestergaard K, Anderson G, Neilsen MI, Jensen TS, Headache in stroke, Stroke, 1993;24:1621-4.

9. Paciaroni M, Parnetti L, Sarchielli P, Gallai V, Headache associated with acute ischemic stroke, I Headache Pain, 2001;2:25-9.

10. Lim L, Merritt's Neurology, Thirteenth Edition, J Neuropathol Exp Neurol, 2016;75:812

11. Headache Classification Committee of the International Headache Society (IHS), The International Classification of Headache Disorders, 3rd edition (beta version), Cephalalgia, 2013:33:629-808

12. Abraham MK, Chang W-TW, Subarachnoid Hemorrhage, Emers Med Clin N Am, 2016;34:901-16.

13. Polmear $A$, Sentinel headaches in aneurysmal subarachnoid haemorrhage: what is the true incidence? A systematic review, Cephalalgia, 2003;23:935-41.

14. Karr SB, Uppin MS, Rajesh A, et al., Vascular malformations of central nervous system: A series from tertiary care hospital in South India, J Neurosci Rural Pract, 2016:7:262-8.

15. Sattar A, Manousakis G, Jensen MB, Systematic review of reversible cerebral vasoconstriction syndrome. Expert Rev Cardiovasc Ther, 2014;8:1417-21.

16. Calabrese LH, Dodick DW, Schwedt TJ, Singhal AB, Narrative review: reversible cerebral vasoconstriction syndromes, Ann Intern Med, 2007;146:34-44.

17. Singhal $A B$, Reversible cerebral vasoconstriction syndromes, Arch Neurol, 2011;68:1005-8.

18. Chen S-P, Fuh J-L, Wang S-J, Reversible cerebral vasoconstriction syndrome: current and future perspectives, Expert Rev Neurother, 2014;11:1265-76
19. Sheikh HU, Mathew PG, Reversible cerebral vasoconstriction syndrome: updates and new perspectives, Curr Pain Headache Rep, 2014:18:414-6.

20. Lee W-J, Jung K-H, Moon J, et al., Prognosis of spontaneous cervical artery dissection and transcranial Doppler findings associated with clinical outcomes, Eur Radiol, 2015;26:1284-91

21. Moon K, Albuquerque FC, Cole T, Stroke prevention by endovascular treatment of carotid and vertebral artery dissections, J Neurointerv Surg, 2016, Epub ahead of print. DOI: 10.1136/neurintsurg-2016-012565.

22. Ortiz I, Ruland $\mathrm{S}$, Cervicocerebral artery dissection, Curr Opin Cardiol, 2015;30:603-10

23. Nouh A, Remke J, Ruland S, Ischemic posterior circulation stroke: a review of anatomy, clinical presentations, diagnosis, and current management, Front Neurol, 2014;5:30.

24. Fusco MR, Harrigan MR, Cerebrovascular dissections - a review, Part I: spontaneous dissections, Neurosurgery, 2011:68:242-57.

25. The CADISS Trial Investigators, Antiplatelet therapy vs. anticoagulation in cervical artery dissection: rationale and design of the Cervical Artery Dissection in Stroke Study (CADISS), Int I Stroke, 2007:2:292-6.

26. Coutinho JM, Cerebral venous thrombosis, J Thromb Haemost 2015; 13:S238-44

27. Stam J, Thrombosis of the cerebral veins and sinuses, N Eng J Med, 2005;352:1791-8.

28. Cumurciuc R, Headache as the only neurological sign of cerebra venous thrombosis: a series of 17 cases, I Neurol Neurosurg Psychiatry, 2005:76:1084-7.

29. Sparaco M, Feleppa M, Bigal ME, Cerebral venous thrombosis and headache - a case-series, Headache, 2015;55:806-14.

30. Einhäupl K, Stam J, Bousser MG, et al., EFNS guideline on the treatment of cerebral venous and sinus thrombosis in adult patients, Eur J Neurol, 2010;17:1229-35.

31. Rykken JB, McKinney AM, Posterior Reversible Encephalopathy Syndrome, Seminars in Ultrasound, CT and MRI, 2014:35:118-35.

32. Fugate JE, Claassen DO, Cloft HJ, Posterior reversible encephalopathy syndrome: associated clinical and radiologic findings, Mayo Clin Proc, 2010;85:427-32.

33. Lamy C, Oppenheim C, Mas JL, Posterior reversible encephalopathy syndrome, Handb Clin Neurol, 2014;121:1687-701

34. Nocton JJ, Usual and unusual manifestations of systemic and central nervous system vasculitis, Pediatr Clin North Am, 2017;64:185-204.

35. Berlit P, Review: Diagnosis and treatment of cerebral vasculitis, Ther Adv Neurol Disord, 2010;3:29-42.
36. Buttgereit F, Dejaco C, Matteson EL, Dasgupta B, Polymyalgia reumatica and giant cell arteritis, JAMA, 2016;315:2442.

37. Delaney JS, Abuzeyad F, Correa JA, Foxford R, Recognition and characteristics of concussions in the emergency department population. J Emerg Med, 2005;29:189-97.

38. Tabatabai RR, Swadron SP, Headache in the emergency department, Emerg Med Clin North Am, 2016;34:695-716.

39. Kirby S, Purdy RA, Headaches and brain tumors, Neurol Clin 2014:32:423-32

40. Wall $\mathrm{M}$, The headache profile of idiopathic intracranial hypertension, Cephalalgia, 1990;10:331-5.

41. Panikkath R, Karukote A, Ruthirago D, et al., Idiopathic intracranial hypertension: ongoing clinical challenges and future prospects, I Pain Res, 2016;9:87-99

42. Pattichis AA, Slee M, CSF hypotension: A review of its manifestations, investigation and management, I Clin NeurosCi, 2016:34:39-43

43. Mokri B, Atkinson JLD, Piepgras DG, Absent headache despite CSF volume depletion (intracranial hypotension), Neurology, 2000;55:1722-4.

44. Ahn JS, Akincioglu C, Gulka I, Coome, G, Spontaneous low cerebrospinal fluid pressure headache: an emergency medicine perspective, CJEM, 2015;15:53-8.

45. Ocak T, Tekin $\mathrm{E}$, Basturk M, et al., Treatment in carbon monoxide poisoning patients with headache: a prospective, multicenter, double-blind, controlled clinical trial, Am J Emerg Med, 2016;34:2140-5.

46. Kristoffersen ES, Lundqvist C, Medication-overuse headache: review, J Pain Res, 2014;7:367-78.

47. Adeney KL, Williams MA, Miller RS, et al., Risk of preeclampsia in relation to maternal history of migraine headaches, J Matern Fetal Neonatal Med, 2005;18:167-72

48. Gelfand A, Goadsby PJ, A neurologist's guide to acute migraine therapy in the emergency room, Neurohospitalist, 2012;2:51-9.

49. Orr SL, Friedman BW, Christie S, et al., Management of adults with acute migraine in the emergency department: The American Headache Society evidence assessment of parenteral pharmacotherapies, Headache, 2016;56:911-40.

50. Lucas $\mathrm{S}$, Medication use in the treatment of migraine during pregnancy and lactation, Curr Pain Headache Rep, 2009;13:392-8

51. Liew Z, Ritz B, Rebordosa C, et al., Acetaminophen use during pregnancy, behavioral problems, and hyperkinetic disorders, IAMA Pediatr, 2014:168:313-20.

52. MacGregor EA, Headache in pregnancy, Continuum (Minneap Minn), 2014;20:128-47 